\title{
Fatores associados à hipertensão arterial: uma revisão sistemática
}

\author{
Factors associated with arterial hypertension: a systematic review
}

Aline Pinto Marques (https://orcid.org/0000-0002-9072-5333) ${ }^{1}$

Célia Landmann Szwarcwald (https://orcid.org/0000-0002-7798-2095) ${ }^{1}$

Débora Castanheira Pires (https://orcid.org/0000-0001-6995-1259) ${ }^{1}$

Jéssica Muzy Rodrigues (https://orcid.org/0000-0003-2526-2317) ${ }^{1}$

Wanessa da Silva de Almeida (https://orcid.org/0000-0002-5164-8603) ${ }^{1}$

Dalia Romero (https://orcid.org/0000-0002-2643-9797) ${ }^{1}$
${ }^{1}$ Laboratório de Informação em Saúde, Instituto de Comunicação e Informação Científica e Tecnológica em Saúde, Fundação Oswaldo Cruz. Av. Brasil 4365, Manguinhos. 21040-900 Rio de Janeiro RJ Brasil. aline.marques@ icict.fiocruz.br

\begin{abstract}
A systematic review of demographic, socioeconomic, behavioral and anthropometric factors associated with hypertension. We included observational studies, of populations aged 18 or older, from the past ten years, published in English, Portuguese or Spanish from Pubmed, Web of Science, Scopus and Lilacs bases. The most found factors related to the greater chance of having hypertension were age and the Body Mass Index (BMI). Other factors associated with the disease were: gender (male), education (lower education), income (lower income) and waist circumference (high). Never having smoked, never having consumed alcohol and white skin color were characteristics related to a lower chance of having hypertension. As demonstrated, demographic, socioeconomic, behavioral and anthropometric characteristics are important factors associated with a greater chance of having hypertension in the adult population. However, while most of the factors associated with it are amenable to intervention, broader health promotion policies will be needed to reduce socioeconomic inequalities in the prevalence of the disease.
\end{abstract}

Key words Risk factors, Hypertension, Review
Resumo Revisão sistemática sobre fatores demográficos, socioeconômicos, comportamentais e antropométricos associados à hipertensão (HAS). Foram incluídos estudos observacionais, de populações com 18 anos ou mais, dos últimos dez anos, publicados em inglês, português ou espanhol das bases Pubmed, Web of Science, Scopus e Lilacs. Ao final, foram incluídos 42 artigos, a maioria do tipo seccional. A idade e o Índice de Massa Corporal (IMC) elevados foram os fatores relacionados à maior chance de ter HAS mais encontrados nos estudos. Outros fatores associados à HAS foram: sexo (masculino), escolaridade (menor escolaridade), renda (menor renda) e circunferência da cintura (elevada). Nunca ter fumado, nunca ter consumido álcool e ter cor branca apareceram como características relacionadas a menor chance de ter HAS. Assim, características demográfcas, socioeconômicas, comportamentais e antropométricas são importantes fatores associados a maior chance de ter HAS na população adulta. Entretanto, enquanto a maioria dos fatores associados à HAS for passivel de intervenção, serão necessárias políticas de promoção da saúde mais amplas para reduzir as desigualdades socioeconômicas na prevalência de hipertensão.

Palavras-chave Fatores de risco, Hipertensão, Revisão 


\section{Introdução}

A hipertensão arterial sistêmica (HAS) é o maior fator de risco para mortalidade no mundo. Estima-se que em 2008, 12,8\% dos óbitos foram decorrentes da $\mathrm{HAS}^{1}$. A HAS contribuiu nas últimas décadas para o aumento da carga de cardiopatias, acidentes cerebrovasculares, insuficiência renal e para as incapacidades prematuras ${ }^{2}$.

Segundo estimativas da Organização Mundial de Saúde (OMS), em 2014, 22,3\% da população mundial com 18 anos ou mais sofria de HAS. Observam-se desigualdades entre os países do mundo, pois enquanto nos países de baixa renda a prevalência de pessoas acometidas pela HAS foi de $27,6 \%$, nos países de alta renda foi de apenas $18,5 \%{ }^{3}$.

Os estudos sobre Carga Global da Doença (GBD) apontam que a HAS aumentou substancialmente entre 1990 e 2015, assim como os óbitos e os anos de vidas perdidos por alguma incapacidade associada à HAS. O último GBD de 2015 destacou que todos os fatores de risco estudados, juntos, foram responsáveis por $57,8 \%$ (IC95\% 56,6-58,8) dos óbitos e 41,2\% dos anos de vida perdidos por incapacidade (DALY). A HAS foi o fator de risco que mais contribuiu para do DALY mundial, superando tabagismo e obesidade $^{4}$.

A prevalência de HAS sofre influência de múltiplos fatores, com destaque para os demográficos, hereditários, socioeconômicos, comportamentais e antropométricos. A maioria desses fatores podem ser controlado ou modificado, sendo então possível reduzir a incidência da hipertensão e de suas complicações ${ }^{2}$. Assim, identificar os fatores associados, bem como conhecer a sua magnitude, constitui elemento de fundamental importância para subsidiar ações de controle da HAS.

Nesse sentido, o objetivo desse estudo foi identificar, por meio de revisão sistemática da literatura, os principais fatores epidemiológicos associados à HAS.

\section{Métodos}

A revisão sistemática utilizou instrumento de coleta previamente formulado e registrado no PROSPERO $^{5}$, base internacional de revisões sistemáticas em saúde e assistência social do Centre for Reviews and Dissemination da Universidade de York. O formulário para extração das informações continha os seguintes itens: a referên- cia completa do artigo, o país de publicação, o período da busca, o desenho de estudo, o local (país/cidade) do estudo, as variáveis analisadas, o método estatístico, os resultados encontrados para a associação entre as variáveis analisadas e a prevalência de HAS e a avaliação da qualidade metodológica dos estudos selecionados.

Os critérios de elegibilidade dos estudos foram: estudos observacionais com objetivo de determinar a associação entre HAS e os fatores demográficos, socioeconômicos, antropométricos e de estilo de vida; estudos cujas associações foram estimadas por meio da utilização de modelos de regressão ajustados; e que utilizaram critérios de seleção da população de estudo adequada ao delineamento proposto (seccional, coorte ou casocontrole). Foram excluídos aqueles que não descreveram o método de análise estatística ou que não utilizaram modelos de regressão ajustados, assim como aqueles que lidaram com populações restritas como gestantes ou doentes crônicos.

Estabelecidos os critérios de inclusão e exclusão e após registro do formulário de extração dos dados procedeu-se à busca nas bases bibliográficas de acesso online Pubmed, Lilacs, Scopus e Web of Science (WOS). Foram selecionados os artigos publicados nos últimos 10 anos, em inglês, espanhol ou português.

$\mathrm{Na}$ estratégia de busca nas bases bibliográficas com vocabulário controlado, Pubmed $(\mathrm{MeSH}$ terms) e Lilacs (DeCS terms), utilizou-se a combinação dos termos "Hypertension/epidemiology" e "risk factor", excluindo-se uma série de descritores referentes a termos diversos daqueles definidos por critérios de pesquisa: "lung", "pulmonary", "intracranial", "ocular", "european continental ancestry group", "white coat syndrome", "cerebrum", "brain", "genes", "adolecent", "infant newborn", "fetus", "proteins", "pragnancy", e "newborn".

Estratégias equivalentes foram utilizadas no processo de busca nas bases bibliográficas Web of Science e Scopus que não usam vocabulário controlado. As duplicidades foram identificadas e excluídas em todas as bases por meio do programa EndNote X 7.0.1.

As buscas eletrônicas foram realizadas por dois revisores independentes ( $1^{\text {a }}$ etapa de seleção). Posteriormente procedeu-se à avaliação da adequabilidade dos títulos ao objetivo do estudo ( $2^{\text {a }}$ etapa de seleção). Os resumos então foram lidos e avaliados ( $3^{a}$ etapa de seleção). Todos os artigos cujos resumos se adequaram aos critérios da pesquisa foram selecionados para leitura na íntegra ( $4^{a}$ etapa de seleção). Os resultados de todas as etapas da seleção foram comparados e 
as discordâncias solucionadas por consenso entre os dois revisores, ou por um terceiro revisor, quando necessário.

A avaliação da qualidade metodológica baseou-se em quatro critérios: seleção adequada das populações de estudo (S); descrição das perdas e sendo esta inferior a $20 \%(\mathrm{P})$; descrição de instrumento de aferição do desfecho (D); e apresentação das estimativas brutas e descrição do processo de seleção das variáveis no modelo ajustado (A). Estes critérios foram propostos por Esteves et al. ${ }^{6}$ com base na Newcastle-Ottawa $\mathrm{Scale}^{7}$, para avaliação de estudos de coorte e nas diretrizes $\mathrm{STROBE}^{8}$ para estudos seccionais.

As variáveis associadas à hipertensão foram classificadas como variáveis demográficas, socioeconômicas, antropométricas e de estilo de vida, a fim de facilitar a análise e comparabilidade dos estudos.

Nesta revisão sistemática, a divulgação dos resultados foi feita com base as diretrizes propostas pela Preferred Reporting Items for Systematic Reviews (PRISMA).

\section{Resultados}

De acordo com a busca eletrônica foram encontradas 5.236 referências, das quais 62 foram excluídas por duplicidade. Após leitura do título excluíram-se 4.979 referências por não atenderem ao objetivo da revisão. Um total de 195 resumos foram avaliados quanto à adequação aos critérios pré-estabelecidos, sendo 130 eliminados. Procedeu-se então à leitura completa dos 65 artigos restantes, dos quais 23 foram excluídos pelas seguintes razões: não realizaram análises ajustadas (oito); não apresentaram seleção adequada da amostra (oito); estudos com populações restritas (cinco); artigo de revisão (um); não apresentou a descrição da metodologia (um). Ao final de todo o processo foram selecionados para inclusão nesta revisão $42 \operatorname{artigos}^{10-51}$ (Figura 1).

A Tabela 1 apresenta as características dos artigos selecionados. A maioria dos estudos foi realizado na Ásia (dezessete $)^{10,12,17,19,21,23,24,28,30,31,33,35,36,38,40,41,45}$, doze na América do Sul ${ }^{13,14,18,22,32,39,44,46-49,51}$, seis na América no Norte $\mathrm{e}^{15,16,29,37,42,43}$, três na Europa ${ }^{26,27,34}$ e quatro na África $^{11,20,25,50}$.

Grande parte dos estudos utilizou a aferição da pressão no momento da entrevista para definir hipertensão (trinta e um) ${ }^{10-13,16-31,33,35,36,38-41,44,45,49,50}$, a autodeclaração de diagnóstico foi utilizada em

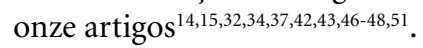

A maioria dos estudos foi do tipo seccional (vinte e nove) ${ }^{10,11,13,14,18-22,24,25,27-}$ $29,31,32,35,36,39,40,42-48,50,51$, onze foram estudos de coorte $\mathrm{e}^{12,15-17,23,26,33,34,37,41,49}$ e apenas dois foram do tipo caso-controle ${ }^{30,38}$. Entre os estudos seccionais, a maior amostra foi de 112.288 indivíduos $^{10} \mathrm{e}$ a menor de $536^{25}$. Nos estudos de coorte a maior amostra compreendeu 42.303 participantes ${ }^{37}$ e a menor $745^{41}$. Em relação aos dois estudos de caso-controle, um foi realizado com base em amostra composta por 700 participantes $^{38}$ e o outro com amostra de apenas 72 participantes ${ }^{30}$.

O modelo de regressão logística foi utilizado em cerca de $80 \%$ das análises, 31 artigos $^{10,11,14,16,17,19-22,24-31,33,35,36,38-43,45,46,48-50}$. O modelo de Cox foi utilizado em cinco artigos ${ }^{12,15,23,34,37}$, o de Poisson em cinco artigos ${ }^{13,18,44,47,51}$ e o modelo linear foi empregado em apenas um artigo ${ }^{32}$.

Todos os estudos foram realizados com base em amostra composta por indivíduos com 18 anos ou mais, no entanto, as faixas etárias estabelecidas em cada artigo foram muito variadas. Vinte faixas etárias foram identificadas.

Em relação à qualidade metodológica, vinte e cinco dos quarenta e dois artigos atingiram a pontuação máxima ${ }^{11-14,16-18,20-23,27-30,33,36-38,40-42,44,50,51}$. Quatorze perderam pontos por não descreverem as perdas $(P)^{10,15,19,24,26,31,32,34,35,39,46-49}$, cinco por não apresentarem as estimativas brutas ou descrição do modelo de análise estatística $(\mathrm{A})^{19,31,43,45,48}$ e um artigo por não descrever o instrumento de aferição do desfecho (D) ${ }^{25}$.

O Quadro 1 apresenta as variáveis demográficas, socioeconômicas, antropométricas e de estilo de vida encontradas nos estudos, o tipo de variável (dicotômica, nominal ou ordinal) e sua associação ou não com o desfecho hipertensão (maior chance, menor chance ou não associação).

As variáveis demográficas associadas à hipertensão foram idade (dezenove artigos), sexo (dezessete artigos), raça ou cor (sete artigos) e área de residência (seis artigos). Em apenas um artigo a variável idade não foi analisada de forma ordinal ${ }^{44}$. Em apenas dois artigos a idade não se mostrou associada ${ }^{42,47}$. Nos demais artigos em que a idade foi considerada, observa-se um aumento progressivo da chance de ter HAS nos indivíduos com idade a partir dos $40 \operatorname{anos}^{10,13,14,18-}$ $21,25,28,29,31,33,39,44-46,50$.

O sexo masculino representou maior chance de ter HAS em nove de dezessete artigos nos quais a variável sexo foi incluída ${ }^{10,13,19,21,25,33,39,42,45}$. A variável cor branca foi estudada de forma dicotômica em quatro artigos ${ }^{18,20,42,46}$ e nominal 


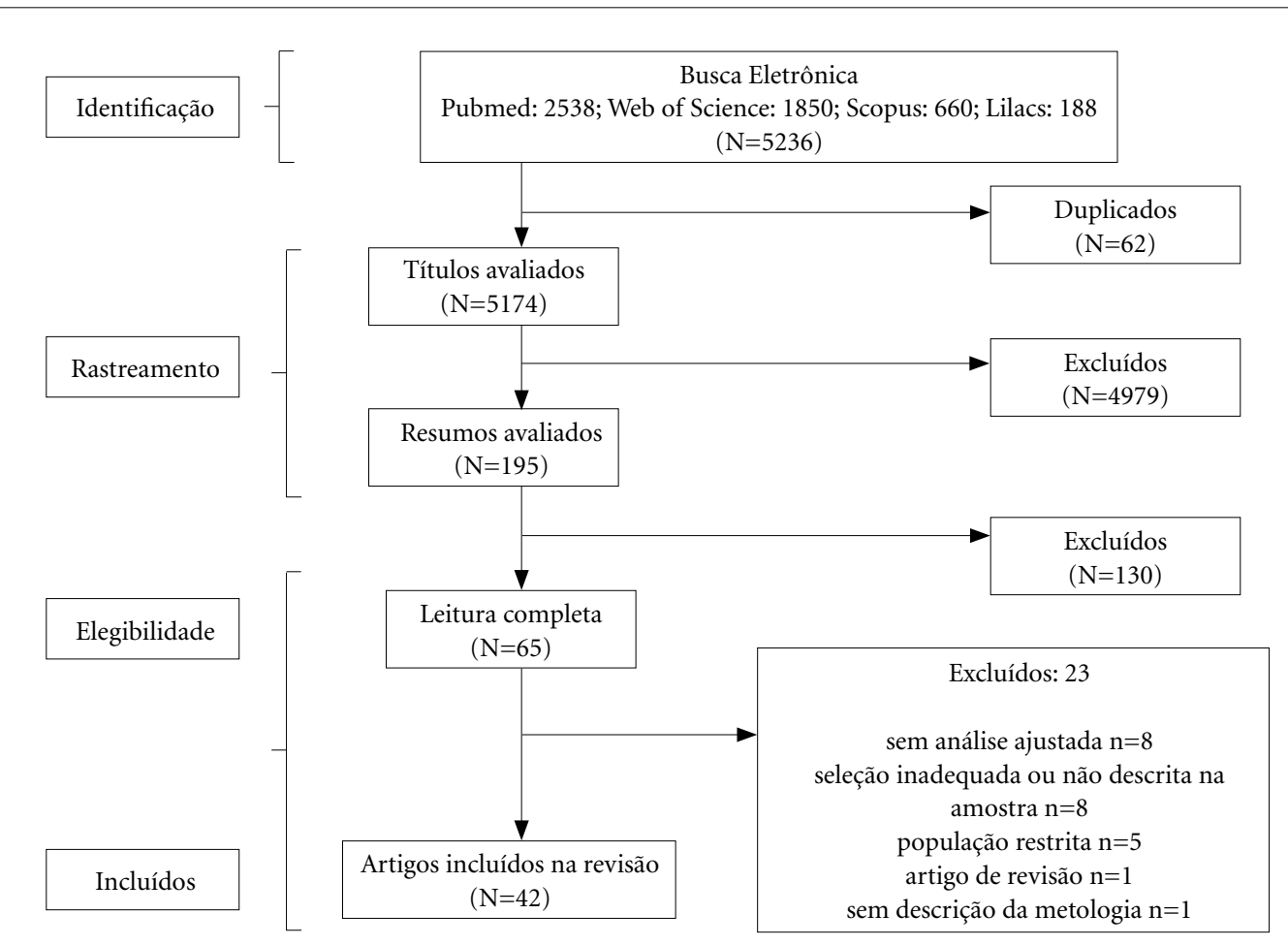

Figura 1. Fluxograma do processo de seleção dos estudos incluídos na revisão sistemática.

em três ${ }^{29,39,43}$. Indivíduos da cor branca apresentaram menor chance de ter HAS em quatro $\operatorname{artigos}^{18,29,43,46}$, enquanto outros três não encontraram associação com raça/cor ${ }^{20,39,42}$. Etnia foi estudada em três artigos, em dois deles essa variável mostrou associação com o desfecho ${ }^{31,45}$. Situação de residência (urbana/rural) foi considerada em seis artigos, em apenas dois residir em área urbana foi associado ao desfecho ${ }^{10,44}$, nos demais a situação de residência não foi associada ${ }^{11,21,31,33}$.

Entre as variáveis socioeconômicas, a mais utilizada foi o grau de escolaridade, incluída em dezoito artigos ${ }^{10,12,14,18,24,25,28-31,33,42-46,50,51}$. Dois artigos analisaram a escolaridade de forma dicotômica $^{30,31}$ e os demais de forma ordinal. O baixo nível de instrução foi associado com a maior chance de ter HAS em dez trabalhos ${ }^{12,25,30,31,44-46,51}$.

A variável estado civil foi considerada em sete artigos, sendo estudada de forma dicotômica em três ${ }^{20,21,50}$ e nominal em quatro ${ }^{14,28,42,44}$. Os resultados encontrados foram diversos, em três artigos, ser casado foi relacionado a menor chance de ter HAS em relação a pelo menos uma outra categoria de estudo ${ }^{20,21,28}$, mas nos outros, a associação com o desfecho não foi observada ${ }^{14,42,44,50}$.
A renda foi analisada de forma ordinal em sete dos oito artigos que utilizaram a variável. Ter menor renda foi associado a maior chance de ter HAS em três estudos ${ }^{18,19,39}$, menor chance em $u^{24}$ e não associado ao desfecho em quatro ${ }^{12,33,42,44}$. Três estudos utilizaram a posse de plano de saúde como variável ${ }^{39,42,51}$, mas nenhuma associação foi encontrada.

A ocupação foi analisada em dois artigos ${ }^{30,39}$. Ser trabalhador manual/agricultor ou desempregado mostrou maior chance de ter HAS nos dois estudos.

As duas medidas antropométricas mais utilizadas nos estudos selecionados foram o índice de massa corporal (IMC) e a circunferência de cintura (CC). O IMC foi considerado na análise de vinte e sete artigos ${ }^{10,13,17-23,25,28-35,39,42,44-50}$, sendo incluído como variável ordinal em vinte e um deles ${ }^{10,13,17,18,20-23,25,28-31,33-35,39,42,44,45,50}$. A CC foi incluída em oito estudos ${ }^{17,27,28,33,39,45,48,49}$, sendo sete como variável dicotômica ${ }^{27,28,33,39,45,47,49}$ e um como variável ordinal ${ }^{17}$. Em todos os artigos ter maior nível de IMC resultou em maior chance de ter HAS, sendo este mais elevado nas categorias de IMC acima de 30, utilizadas para caracterizar 
Tabela 1. Características dos estudos incluídos na revisão sistemática.

\begin{tabular}{|c|c|c|c|c|c|c|c|c|c|}
\hline \multirow{2}{*}{$\begin{array}{l}\text { Autor (ano de } \\
\text { publicação) }\end{array}$} & \multirow{2}{*}{$\begin{array}{l}\text { Definição de } \\
\text { hipertensão }\end{array}$} & \multirow[t]{2}{*}{ Cidade/País } & \multirow[t]{2}{*}{ Idade } & \multirow{2}{*}{$\begin{array}{l}\text { Tipo de estudo } \\
\text { (amostra) }\end{array}$} & \multirow{2}{*}{$\begin{array}{c}\text { Modelo } \\
\text { de } \\
\text { regressão }\end{array}$} & \multicolumn{4}{|c|}{$\begin{array}{c}\text { Avaliação } \\
\text { metodológica }\end{array}$} \\
\hline & & & & & & $S$ & $\mathbf{P}$ & $\mathrm{D}$ & A \\
\hline $\begin{array}{l}\text { Abdul-Razak et al. } \\
(2016)^{10}\end{array}$ & Aferida & Malásia & $\geq 30$ anos & $\begin{array}{l}\text { Seccional } \\
(112.288)\end{array}$ & Logística & 1 & 0 & 1 & 1 \\
\hline Abebe et al. $(2015)^{11}$ & Aferida & Etiópia & $\geq 35$ anos & Seccional (2.200) & Logística & 1 & 1 & 1 & 1 \\
\hline Baek et al. $(2015)^{12}$ & Aferida & Coréia do Sul & 40 a 69 anos & Coorte (5.282) & Cox & 1 & 1 & 1 & 1 \\
\hline $\begin{array}{l}\text { Barbosa et al. } \\
(2008)^{13}\end{array}$ & Aferida & São Luís (Brasil) & 18 a 94 anos & Seccional (835) & Poisson & 1 & 1 & 1 & 1 \\
\hline Borges et al. $(2008)^{14}$ & Autorreferência & Belém (Brasil) & $\geq 18$ anos & Seccional (2.352) & Logística & 1 & 1 & 1 & 1 \\
\hline Borgi et al. $(2016)^{15}$ & Autorreferência & EUA & 30 a 75 anos & $\begin{array}{l}\text { Coorte } \\
\left(187.453^{\star}\right)\end{array}$ & Cox & 1 & 0 & 1 & 1 \\
\hline $\begin{array}{l}\text { Carnethon et al. } \\
(2010)^{16}\end{array}$ & Aferida & EUA & 18 a 30 anos & Coorte (4.618) & Logística & 1 & 1 & 1 & 1 \\
\hline Chei et al. $(2008)^{17}$ & Aferida & Japão & 40 a 69 anos & Coorte $(2.790)$ & Logística & 1 & 1 & 1 & 1 \\
\hline Costa et al. $(2007)^{18}$ & Aferida & Pelotas (Brasil) & 20 a 69 anos & Seccional (1.800) & Poisson & 1 & 1 & 1 & 1 \\
\hline Do an et al. $(2012)^{19}$ & Aferida & Turquia & $\geq 18$ anos & Seccional (2.035) & Logística & 1 & 0 & 1 & 0 \\
\hline $\begin{array}{l}\text { Doulougou et al. } \\
(2014)^{20}\end{array}$ & Aferida & $\begin{array}{l}\text { Ouagadougou } \\
\text { (Burkina Faso) }\end{array}$ & $\geq 18$ anos & Seccional (2.041) & Logística & 1 & 1 & 1 & 1 \\
\hline $\begin{array}{l}\text { Esteghamati et al. } \\
(2008)^{21}\end{array}$ & Aferida & Irã & 25 a 64 anos & $\begin{array}{l}\text { Seccional } \\
(70.329)\end{array}$ & Logística & 1 & 1 & 1 & 1 \\
\hline $\begin{array}{l}\text { Ferreira et al. } \\
(2009)^{22}\end{array}$ & Aferida & $\begin{array}{l}\text { Capitais e DF } \\
\text { (Brasil) }\end{array}$ & $\geq 18$ anos & $\begin{array}{l}\text { Seccional } \\
(54.369)\end{array}$ & Logística & 1 & 1 & 1 & 1 \\
\hline Fujita et al. $(2013)^{23}$ & Aferida & Chiba (Japão) & 40 a 79 anos & Coorte (29.604) & Cox & 1 & 1 & 1 & 1 \\
\hline $\begin{array}{l}\text { Harshfield et al. } \\
(2015)^{24}\end{array}$ & Aferida & $\begin{array}{l}\text { Bangladesh } \\
\text { (India) }\end{array}$ & $\geq 35$ anos & Seccional (8.834) & Logística & 1 & 0 & 1 & 1 \\
\hline Helelo et al. $(2014)^{25}$ & Aferida & Etiópia & $\geq 31$ anos & Seccional (536) & Logística & 1 & 1 & 0 & 1 \\
\hline $\begin{array}{l}\text { Heraclides et al. } \\
(2015)^{26}\end{array}$ & Aferida & Reino Unido & 43 a 53 anos & Coorte (1.750) & Logística & 1 & 0 & 1 & 1 \\
\hline Hirani et al. $(2007)^{27}$ & Aferida & Inglaterra & $\geq 35$ anos & $\begin{array}{l}\text { Seccional } \\
(10.890)\end{array}$ & Logística & 1 & 1 & 1 & 1 \\
\hline $\begin{array}{l}\text { Janghorbani et al. } \\
(2008)^{28}\end{array}$ & Aferida & Irã & 25 a 64 anos & $\begin{array}{l}\text { Seccional } \\
(69.722)\end{array}$ & Logística & 1 & 1 & 1 & 1 \\
\hline $\begin{array}{l}\text { Leenen et al. } \\
(2010)^{29}\end{array}$ & Aferida & $\begin{array}{l}\text { Ontario } \\
\text { (Canadá) }\end{array}$ & 20 a 79 anos & Seccional (2.510) & Logística & 1 & 1 & 1 & 1 \\
\hline Lwin et al. $(2013)^{30}$ & Aferida & Tailândia & $\geq 35$ anos & $\begin{array}{l}\text { Caso-controle } \\
(72)\end{array}$ & Logística & 1 & 1 & 1 & 1 \\
\hline $\begin{array}{l}\text { Malekzadeh et al. } \\
(2013)^{31}\end{array}$ & Aferida & Irã & 40 a 75 anos & $\begin{array}{l}\text { Seccional } \\
(50.045)\end{array}$ & Logística & 1 & 0 & 1 & 0 \\
\hline $\begin{array}{l}\text { Muraro et al. } \\
(2013)^{32}\end{array}$ & Autorreferência & $\begin{array}{l}\text { Capitais e DF } \\
\text { (Brasil) }\end{array}$ & $\geq 18$ anos & $\begin{array}{l}\text { Seccional } \\
(54.353)\end{array}$ & Linear & 1 & 0 & 1 & 1 \\
\hline Niu et al. $(2014)^{33}$ & Aferida & China & $\geq 18$ anos & Coorte (6.096) & Logística & 1 & 1 & 1 & 1 \\
\hline $\begin{array}{l}\text { Núñez-Córdoba et } \\
\text { al }(2009)^{34}\end{array}$ & Autorreferência & Espanha & 20 a 90 anos & Coorte (9.408) & Cox & 1 & 0 & 1 & 1 \\
\hline Pang et al. $(2008)^{35}$ & Aferida & $\begin{array}{l}\text { Fuxin County } \\
\text { (China) }\end{array}$ & $\geq 35$ anos & $\begin{array}{l}\text { Seccional } \\
(45.925)\end{array}$ & Logística & 1 & 0 & 1 & 1 \\
\hline $\begin{array}{l}\text { Radhika et al. } \\
(2007)^{36}\end{array}$ & Aferida & Chennai (India) & $\geq 20$ anos & Seccional (1.902) & Logística & 1 & 1 & 1 & 1 \\
\hline Sesso et al. $(2008)^{37}$ & Autorreferência & EUA & $\geq 40$ anos & Coorte (42.303) & Cox & 1 & 1 & 1 & 1 \\
\hline $\begin{array}{l}\text { Shankarishan et al. } \\
(2012)^{38}\end{array}$ & Aferida & $\begin{array}{l}\text { Dibrugarh } \\
\text { (India) }\end{array}$ & 20 a 65 anos & $\begin{array}{l}\text { Caso-controle } \\
(700)\end{array}$ & Logística & 1 & 1 & 1 & 1 \\
\hline Silva et al. $(2012)^{39}$ & Aferida & Brasil & 20 a 59 anos & Seccional (2.016) & Logística & 1 & 0 & 1 & 1 \\
\hline Song et al. $(2014)^{40}$ & Aferida & Coréia do Sul & 19 a 64 anos & Seccional (9.791) & Logística & 1 & 1 & 1 & 1 \\
\hline
\end{tabular}


Tabela 1. Características dos estudos incluídos na revisão sistemática.

\begin{tabular}{|c|c|c|c|c|c|c|c|c|c|}
\hline \multirow{2}{*}{$\begin{array}{l}\text { Autor (ano de } \\
\text { publicação) }\end{array}$} & \multirow{2}{*}{$\begin{array}{l}\text { Definição de } \\
\text { hipertensão }\end{array}$} & \multirow[t]{2}{*}{ Cidade/País } & \multirow[t]{2}{*}{ Idade } & \multirow{2}{*}{$\begin{array}{l}\text { Tipo de estudo } \\
\text { (amostra) }\end{array}$} & \multirow{2}{*}{$\begin{array}{c}\text { Modelo } \\
\text { de } \\
\text { regressão }\end{array}$} & \multicolumn{4}{|c|}{$\begin{array}{c}\text { Avaliação } \\
\text { metodológica }\end{array}$} \\
\hline & & & & & & $S$ & $\mathbf{P}$ & D & $\mathrm{A}$ \\
\hline $\begin{array}{l}\text { Tsubota-Utsugi et al. } \\
(2011)^{41}\end{array}$ & Aferida & $\begin{array}{l}\text { Ohasama } \\
\text { (Japão) }\end{array}$ & $\geq 35$ anos & Coorte (745) & Logística & 1 & 1 & 1 & 1 \\
\hline $\begin{array}{l}\text { Sampson et al. } \\
(2014)^{42}\end{array}$ & Autorreferência & EUA & 40 a 79 anos & $\begin{array}{l}\text { Seccional } \\
(69.211)\end{array}$ & Logística & 1 & 1 & 1 & 1 \\
\hline Veenstra $(2013)^{43}$ & Autorreferência & Canadá & $\geq 25$ anos & $\begin{array}{l}\text { Seccional } \\
(90.310)\end{array}$ & Logística & 1 & 1 & 1 & 0 \\
\hline $\begin{array}{l}\text { Camacho et al. } \\
(2016)^{44}\end{array}$ & Aferida & Colômbia & 35 a 70 anos & Seccional (7.444) & Poisson & 1 & 1 & 1 & 1 \\
\hline Liu et al. $(2017)^{45}$ & Aferida & $\begin{array}{l}\text { Chongqing } \\
\text { Province (China) }\end{array}$ & $\geq 18$ anos & $\begin{array}{l}\text { Seccional } \\
(14.420)\end{array}$ & Logística & 1 & 1 & 1 & 0 \\
\hline Malta et al. $(2017)^{46}$ & Autorreferência & $\begin{array}{l}\text { Capitais e DF } \\
\text { (Brasil) }\end{array}$ & $\geq 18$ anos & $\begin{array}{l}\text { Seccional } \\
(52.929)\end{array}$ & Logística & 1 & 0 & 1 & 1 \\
\hline $\begin{array}{l}\text { Moreira et al. } \\
(2017)^{47}\end{array}$ & Autorreferência & $\begin{array}{l}\text { João Pessoa } \\
\text { (Brasil) }\end{array}$ & $\geq 45$ anos & Seccional (867) & Poisson & 1 & 0 & 1 & 1 \\
\hline $\begin{array}{l}\text { Oliveira et al. } \\
(2017)^{48}\end{array}$ & Autorreferência & Baependi (Brasil) & $\geq 18$ anos & Seccional (1.627) & Logística & 1 & 0 & 1 & 0 \\
\hline Silva et al. $(2017)^{49}$ & Aferida & $\begin{array}{l}\text { Florianópolis } \\
\text { (Brasil) }\end{array}$ & $\geq 18$ anos & Coorte (1.222) & Logística & 1 & 0 & 1 & 1 \\
\hline $\begin{array}{l}\text { Soubeiga at al. } \\
(2017)^{50}\end{array}$ & Aferida & Burkina Faso & 24 a 64 anos & Seccional (4.629) & Logística & 1 & 1 & 1 & 1 \\
\hline Malta et al $(2016)^{51}$ & Autorreferência & Brasil & $\geq 18$ anos & $\begin{array}{l}\text { Seccional } \\
(62.986)\end{array}$ & Poisson & 1 & 1 & 1 & 1 \\
\hline
\end{tabular}

* Número total referente a três coortes independentes.

obesidade. Resultado semelhante foi encontrado para a CC, isto é, quanto maior a medida da CC, maior a chance de ter HAS. Em apenas um estudo a CC não se mostrou associada ao desfecho ${ }^{17}$.

No que se refere às variáveis de estilo de vida, o tabagismo e a atividade física foram as variáveis mais encontradas na revisão. Os autores analisaram o tabagismo de diferentes formas: oito como variável dicotômica ${ }^{11,20,30,33,38,44,45,50}$, quatro como variável nominal ${ }^{10,14,22,28}$ e quatro como variável ordinal ${ }^{18,31,39,42}$. O tabagismo não foi associado ao desfecho na maioria dos arti$\operatorname{gos}^{10,11,14,18,20,28,30,33,39,42,44,50}$. Nunca ter fumado apareceu como relacionado a menor chance de ter HAS em três estudos ${ }^{22,38,45}$ e em um estudo foi relacionado a maior chance de ter $\mathrm{HAS}^{31}$.

Já no que se refere à atividade física, grande parte dos estudos analisou esta variável de forma dicotômica ${ }^{11,16,18,22,30,32,39,44,46,47,50}$. Ter baixa atividade física representou maior chance de ter HAS em quatro artigos ${ }^{20,22,25,39}$ e nos demais não teve associação com $\mathrm{HAS}^{11,16,18,29,30,32,44,46,47,50}$.

Dos onze artigos que incluíram consumo de álcool ${ }^{11,18,20,30,32,33,37,38,42,44,45}$, em sete a variável foi analisada como dicotômica. A variável não apre- sentou associação em sete $\operatorname{artigos}^{11,18,20,30,32,33,44}$. Nos outros quatro, não ter consumido álcool representou menor chance de ter $\mathrm{HAS}^{37,38,42,45}$. A adição de sal à refeição não foi associada à HAS em três estudos ${ }^{18,22,45}$, mas mostrou maior chance de ter HAS em outros três ${ }^{25,36,46}$.

Variáveis referentes ao hábito alimentar foram incluídas em onze artigos, com grande variação entre as características culturais avaliadas. Levando em consideração os dados apresentados pelos autores, foram agrupadas as variações alimentares de maneira dicotômica entre alimentação adequada e inadequada. A alimentação inadequada apresentou maior chance de ter HAS em apenas dois $\operatorname{artigos}^{10,25}$, menor chance em dois ${ }^{15,32}$ e não mostrou associação com o desfecho nos demais $^{14,26,30,31,33,34,40}$.

\section{Discussão}

Idade e IMC elevados foram as variáveis mais frequentemente encontrados nos artigos selecionados na presente revisão. Ambos representaram associação em cerca de metade dos estudos 
Quadro 1. Tipo e associação das variáveis demográficas, socioeconômicas, antropométricas e de estilo de vida com a hipertensão, segundo os artigos revisados.

\begin{tabular}{|c|c|c|c|c|c|c|c|}
\hline \multirow[b]{2}{*}{ Características } & \multicolumn{3}{|c|}{ Tipo de varável } & \multicolumn{3}{|c|}{ Associação } & \multirow{2}{*}{$\begin{array}{c}\text { Número } \\
\text { de } \\
\text { artigos }\end{array}$} \\
\hline & Dicotômica & Nominal & Ordinal & $\begin{array}{l}\text { Maior } \\
\text { chance }\end{array}$ & $\begin{array}{l}\text { Menor } \\
\text { chance }\end{array}$ & $\begin{array}{c}\text { Não } \\
\text { associado }\end{array}$ & \\
\hline \multicolumn{8}{|c|}{ Demográficas } \\
\hline Idade mais elevada & 44 & & \begin{tabular}{|r}
$10,13,14,18$ \\
$19,20,21,25$ \\
$28,29,31,33$ \\
$39,45,42,46$ \\
47,50
\end{tabular} & \begin{tabular}{|r}
$10,13,14,18$ \\
$19,20,21$ \\
$25,28,29,31$ \\
$33,39,44,45$ \\
46,50
\end{tabular} & & 42,47 & 19 \\
\hline Homem & $\begin{array}{r}10,11,13,18, \\
19,21,25,29 \\
31,33,39,42, \\
43,44,45 \\
46,50 \\
\end{array}$ & & & $\begin{array}{r}\mid 10,13,19,21 \\
25,33,39 \\
42,45\end{array}$ & 31 & $\begin{array}{r}11,18,29,43 \\
44,46,50\end{array}$ & 17 \\
\hline Cor branca & $18,20,42,46$ & $29,39,43$ & & & $18,29,43,46$ & $20,39,42$ & 7 \\
\hline Etnia & $10,31,45$ & & & 31,45 & & 10 & 3 \\
\hline $\begin{array}{l}\text { Residir em área } \\
\text { urbana }\end{array}$ & $\begin{array}{r}10,11,21,31 \\
33,44\end{array}$ & & & 44 & 10 & $11,21,31,33$ & 6 \\
\hline \multicolumn{8}{|c|}{ Socioeconômicas } \\
\hline $\begin{array}{l}\text { Menor } \\
\text { escolaridade }\end{array}$ & 30,31 & & \begin{tabular}{|r|}
$10,12,14,18$ \\
$24,25,28,29$ \\
$33,42,43,44$, \\
$45,46,50,51$
\end{tabular} & \begin{tabular}{|r|}
$12(\mathrm{M}), 25$ \\
$30,31,42,43$ \\
$44,45,46,51$
\end{tabular} & & \begin{tabular}{|r|}
$10,12(\mathrm{H})$ \\
$14,18,24,28$, \\
$29,33,50$
\end{tabular} & 18 \\
\hline Casado & $20,21,50$ & $\begin{array}{r}14,28 \\
42,44\end{array}$ & & & $\begin{array}{r}20(\mathrm{M}), 21, \\
28\end{array}$ & $\begin{array}{r}14,20(\mathrm{H}) \\
42,44,50\end{array}$ & 7 \\
\hline Menor renda & 19 & & $\begin{array}{r}12,18,24,33 \\
39,42,44 \\
\end{array}$ & $18,19,39$ & 24 & $12,33,42,44$ & 8 \\
\hline Ter plano de saúde & 39,51 & 42 & & & & $39,42,51$ & 3 \\
\hline $\begin{array}{l}\text { Trabalhador } \\
\text { manual/agricultor } \\
\text { ou desempregado }\end{array}$ & 39 & 30 & & 30,39 & & & 2 \\
\hline \multicolumn{8}{|c|}{ Antropométricas } \\
\hline IMC elevado & $\begin{array}{r}19,32,46,47 \\
48,49\end{array}$ & & \begin{tabular}{|r}
$10,13,17,18$ \\
$20,21,22,23$ \\
$25,28,29,30$ \\
$31,33,34,35$ \\
$39,42,44$ \\
45,50
\end{tabular} & \begin{tabular}{|r|}
$10,13,17,18$ \\
$19,20,21,22$ \\
$23,25,28,29$ \\
$30,31,32,33$ \\
$34,35,39,42$ \\
$44,45,46,47$ \\
$48,49,50$ \\
\end{tabular} & & & 27 \\
\hline $\begin{array}{l}\text { Circunferência da } \\
\text { Cintura elevada }\end{array}$ & $\begin{array}{r}27,28,47,49 \\
33,45,39 \\
\end{array}$ & & 17 & $\begin{array}{r}27,28,33,39 \\
45,48,49 \\
\end{array}$ & & 17 & 8 \\
\hline \multicolumn{8}{|c|}{ Estilo de vida } \\
\hline Nunca fumou & $\begin{array}{l}11,20,30,33, \\
38,44,45,50\end{array}$ & $\begin{array}{r}10,14 \\
22,28\end{array}$ & $18,31,39,42$ & 31 & $22,38,45$ & \begin{tabular}{|c|}
$10,11,14,18$ \\
$20,28,30,33$ \\
$39,42,44,50$
\end{tabular} & 16 \\
\hline $\begin{array}{l}\text { Baixa atividade } \\
\text { física }\end{array}$ & $\begin{array}{r}11,16,18,22, \\
30,32,39,44, \\
46,47,50\end{array}$ & & $20,25,29$ & $20,22,25,39$ & & $\begin{array}{r}11,16,18,29 \\
30,32,44,46, \\
47,50\end{array}$ & 14 \\
\hline $\begin{array}{l}\text { Nunca consumiu } \\
\text { álcool }\end{array}$ & $\begin{array}{r}11,30,32,33 \\
38,44,45\end{array}$ & & $18,20,37,42$ & & $\begin{array}{r}37,38,42 \\
45\end{array}$ & $\begin{array}{r}11,18,20,30 \\
32,33,44\end{array}$ & 11 \\
\hline $\begin{array}{l}\text { Adiciona sal às } \\
\text { refeições }\end{array}$ & $18,22,25,46$ & & 36,45 & $25,36,46$ & & $18,22,45$ & 6 \\
\hline $\begin{array}{l}\text { Alimentação } \\
\text { inadequada ou de } \\
\text { risco }\end{array}$ & $\begin{array}{r}14,15,25 \\
32,33\end{array}$ & & $\begin{array}{r}10,26,30,31 \\
34,40\end{array}$ & 10,25 & 15,32 & $\begin{array}{r}14,26,30,31 \\
33,34,40\end{array}$ & 11 \\
\hline
\end{tabular}

$\mathrm{M}=$ Mulher; $\mathrm{H}=$ Homem. 
analisados. Merecem destaque ainda, as variáveis sexo, escolaridade, renda e CC, onde as categorias "sexo masculino", "menor escolaridade", "menor renda" e "circunferência de cintura elevada" foram associadas à maior chance de ter HAS em pelo menos três artigos. Indivíduos nas categorias "cor branca", "nunca fumou" e "nunca consumiu álcool" apresentaram menor chance de ter HAS em pelo menos três artigos.

A idade elevada é um fator associado HAS já conhecido na literatura especializada. $\mathrm{O}$ envelhecimento traz modificações fisiológicas do aparelho circulatório, aumentado assim a chance de desenvolvimento da $\mathrm{HAS}^{52}$. O aumento da prevalência de idosos no mundo é apontado pelos especialistas como o fator explicativo de maior destaque para o aumento da prevalência de HAS em todo o planeta ${ }^{2,53}$. Dos dezenove estudos nos quais foi considerada na análise, em dezessete apresentou associação direta entre idade elevada e HAS.

Maior chance de ter HAS entre os homens foi encontrada em alguns artigos. Segundo a OMS, em análise referente ao ano de 2008, os homens apresentaram maior prevalência de HAS em todas as regiões do mundo ${ }^{1}$. No Brasil, estudos baseados em informações de diagnóstico médico autorreferido mostram que a prevalência de HAS em mulheres foi mais alta ${ }^{54,55}$. Resultado semelhante foi observado na Pesquisa Nacional de Saúde (PNS) de 2013, onde a prevalência autorreferida de HAS foi maior entre as mulheres. Contudo, cabe ressaltar que quando foram consideradas as aferições da pressão arterial, a HAS foi mais prevalente entre os homens ${ }^{56}$. Este fato reforça o argumento de que a maior utilização dos serviços de saúde explicaria a maior prevalência de HAS autorreferida entre as mulheres ${ }^{57}$.

Ter cor branca apresentou menor chance de ter HAS em quatro artigos analisados. Alguns estudos apontam que indivíduos não brancos apresentam maior chance de desenvolver hipertensão por características genéticas ${ }^{58,59}$. No entanto, não existe consenso sobre esse argumento ${ }^{60}$. Ao analisar a relação entre raça/cor com HAS é preciso levar em consideração fatores do contexto histórico, econômico e político-social ${ }^{61}$, uma vez que pessoas de cor negra têm, em geral, maior vulnerabilidade social.

Entre as variáveis socioeconômicas mais encontradas destacaram-se a escolaridade e a renda. Quanto menor a escolaridade e a renda, maior a chance de ter HAS. Estudos apontam que tanto características socioeconômicas do nível individual quanto macroeconômicas estão associadas à maior chance de ter HAS em pessoas e populações de menor status socioeconômico. Destacam que indivíduos expostos às condições de fragilidade social e econômica, individuais ou das áreas nas quais residem, têm maior chance de desenvolver $\mathrm{HAS}^{62,63}$. No entanto, discutem que os mecanismos subjacentes a esta associação ainda precisam ser melhor explorados.

A obesidade apareceu como o principal fator antropométrico associado a HAS incluído nos artigos selecionados. De fato, a obesidade é um forte preditor das doenças cardiovasculares ${ }^{2}$. Esse resultado torna-se ainda mais preocupante quando consideramos que o Brasil é o quinto país com maior prevalência de obesos ${ }^{64}$, com $21 \%$ de obesos na população com mais de 18 anos em $2013^{65}$.

Assim como a obesidade, a falta de atividade física regular aumenta a chance de doenças cardiovasculares e sua relação com maior prevalência de HAS é conhecida na literatura ${ }^{2}$. Nesta revisão, ser fisicamente inativo representou maior chance de ter HAS em quatro artigos.

O tabagismo e o consumo de álcool são fatores de risco para doenças cardiovasculares conhecidos na literatura. Os efeitos do tabaco e álcool no sistema circulatório são nocivos aumentando o risco de $\mathrm{HAS}^{66,67}$. Os avanços dos estudos epidemiológicos mostraram que embora a baixa ingestão de bebidas alcoólicas leves possa ser benéfica à saúde, o consumo abusivo de álcool está diretamente associado à maior mortalidade cardiovascular ${ }^{66}$.

O tabagismo aumenta a pressão arterial, dobra o risco de desenvolvimento da HAS e provoca uma maior variabilidade nos níveis pressóricos, podendo causar lesões nos órgãos-alvo ${ }^{68}$. Além disso, um estudo sobre carga global da doença apontou que o tabagismo é responsável por $45 \% \mathrm{e}$ $25 \%$ dos óbitos por infarto agudo do miocárdio e por doenças cerebrovasculares, respectivamente ${ }^{69}$.

O método de definição da HAS variou entre aferições realizadas pelos estudos e autodeclaração de diagnóstico prévio. Grande parte dos que usaram aferição eram estudos internacionais, no Brasil, cinco estudos utilizaram a aferição da pressão arterial para definir os indivíduos hipertensos. O uso da aferição é um método mais robusto, uma vez que não está influenciado por fatores como acesso a serviços de saúde e memória do participante da pesquisa. Contudo, pelo menos desde a década de 1990, estudos conduzidos em diversos mostram que a hipertensão arterial autorreferida tem alta sensibilidade, especificidade e bons valores preditivos (positivos e negativos $)^{70}$. 
O presente estudo apresenta algumas limitações, a primeira diz respeito a estratégia de busca. Embora tenha sido feita de forma a abarcar o maior número possível de publicações, pode-se ter deixado de captar trabalhos relevantes por falhas na estratégia de busca por palavras chaves ou descritores. Outra limitação encontrada foi a impossibilidade de realizar a síntese dos resultados por meio de meta-análise, uma vez que a heterogeneidade demográfica, regional e cultural dos estudos reduziu sua comparabilidade.

Os fatores associados à HAS são multidimensionais e seu debate em todo o mundo ainda precisa de aprofundamento. Embora haja consenso entre os estudos acerca dos limites pressóricos para considerar um indivíduo hipertenso, o que garante comparabilidade, não há consenso entre os estudos sobre a seleção e categorização dos fatores associados à HAS. Além disso, há grande variação entre os efeitos desses fatores e sobre quais se mostraram associados à HAS.

Espera-se que os resultados desse trabalho contribuam para o debate sobre os fatores associados à HAS. O rápido processo de envelhecimento alerta para necessidade de programas voltados para o controle da HAS, especialmente entre indivíduos com 40 anos ou mais, cuja chance de desenvolver HAS nesse estudo mostraramse crescente com a idade. A promoção de hábitos de vida saudáveis, como por exemplo prática de atividade regular e controle do peso corporal, aparecem como ações estratégicas para o combate da obesidade que apresenta tendência crescente e está altamente associada a HAS.

Outro aspecto importante foi renda e grau de escolaridade baixos destacados por esta revisão, indicando que esforços para a ampliação das políticas de promoção da saúde nas classes em desvantagem social são necessários para a redução das desigualdades socioeconômicas relacionadas à ocorrência de hipertensão ${ }^{71}$. Tendo em vista que a HAS é um agravo silencioso e, portanto, diagnosticado e tratado com mais frequência entre aqueles com maior acesso à assistência de saúde, a diminuição dos fatores associados nos grupos de baixo nível socioeconômico contribuiria não apenas para a diminuição da incidência de HAS na população, mas também para a diminuição de cardiopatias, acidentes cerebrovasculares, e outras doenças relacionadas à hipertensão ${ }^{72}$.

Destaca-se, assim, nessa revisão a importância de considerar os fatores passíveis de intervenção, e enfatizar a necessidade de políticas de promoção à saúde mais amplas para reduzir as desigualdades socioeconômicas e a da incidência de hipertensão. Consequentemente, acarretaria a redução das doenças associadas à hipertensão e das limitações e incapacidades, implicando, por sua vez, no aumento da esperança de vida saudável nas classes em desvantagem social ${ }^{73}$.

\section{Colaboradores}

Todos os autores contribuíram igualmente em todas as etapas do desenvolvimento deste trabalho. 


\section{Referências}

1. World Health Organization (WHO). Global health risks: mortality and burden of disease attributable to selected major risks. Geneva: WHO; 2009.

2. Organización Mundial de la Salud (OMS). Información general sobre la hipertensión en el mundo. Una enfermedad que mata en silencio, una crisis de salud pública mundial. Día Mundial de la Salud 2013. Ginebra: OMS; 2013.

3. World Health Organization (WHO). Indicator and Measurement Registry [Internet] [acessado 2016 Jun 10]. Disponível em: http://apps.who.int/gho/indicatorregistry/App_Main/browse_indicators.aspx. Acesso em: 10 de junho de 2016

4. Murray CJ, Forouzanfar MH, Liu P, Roth GA, Ng M, Biryukov S, Marczak L, et al. Global Burden of Hypertension and Systolic Blood Pressure of at Least 110 to 115 mm Hg, 1990-2015. JAMA 2017; 317(2):165-182.

5. National Institute for Health Research (NHS). University of York. PROSPERO: International prospective register of systematic reviews [Internet]. [acessado 2016 Jun 10]. Disponível em: http://www.crd.york. ac.uk/PROSPERO/

6. Esteves TMB, Daumas RP, Oliveira MIC, Andrade CAF. Fatores associados à amamentação na primeira hora de vida: revisão sistemática. Rev Saude Publica 2014; 48(4):697-708.

7. Stang A. Critical evaluation of the Newcastle-Ottawa scale for the assessment of the quality of nonrandomized studies in meta-analyses. Eur J Epidemiol 2010; 25(9):603-605.

8. Malta M, Cardoso LO, Bastos FI, Magnanini MM, Silva CM. STROBE initiative: guidelines on reporting observational studies. Rev Saude Publica 2010; 44(3):559-565.

9. Liberati A, Altman DG, Tetzlaff J, Mulrow C, Gotzsche PC, Ioannidis JP, Clarke M, Devereaux PJ, Kleijnen J, Moher D. The PRISMA statement for reporting systematic reviews and meta-analyses of studies that evaluate healthcare interventions: explanation and elaboration. BMJ 2009; 339:b2700.

10. Abdul-Razak S, Daher AM, Ramli AS, Ariffin F, Mazapuspavina MY, Ambigga KS, Miskan M, Abdul -Hamid H, Mat-Nasir N, Nor-Ashikin MN, Ng KK, Nawawi H, Yusoff K, REDISCOVER Investigators. Prevalence, awareness, treatment, control and socio demographic determinants of hypertension in Malaysian adults. BMC Public Health 2016; 16:351.

11. Abebe SM, Berhane Y, Worku A, Getachew A. Prevalence and associated factors of hypertension: a crossectional community based study in Northwest Ethiopia. Plos One 2015; 10(4):e0125210.

12. Baek TH, Lee HY, Lim NK, Park HY. Gender differences in the association between socioeconomic status and hypertension incidence: the Korean Genome and Epidemiology Study (KoGES). BMC Public Health 2015; 15:852.

13. Barbosa JB, Silva AAM, Santos AM, Monteiro Júnior FC, Barbosa MM, Barbosa MM, Figueiredo Neto JA, Soares NJS, Nina VJS, Barbosa JN. Prevalência da hipertensão arterial em adultos e fatores associados em São Luís - MA. Arq Bras Cardiol 2008; 91(4):260-266.
14. Borges HP, Cruz NC, Moura EC. Associação entre hipertensão arterial e excesso de peso em adultos, Belém, Pará, 2005. Arq Bras Cardiol 2008; 91(2):110-118.

15. Borgi L, Muraki I, Satija A, Willett WC, Rimm EB, Forman JP. Fruit and vegetable consumption and the incidence of hypertension in three prospective cohort studies. Hypertension 2016; 67(2):288-293.

16. Carnethon MR, Evans NS, Church TS, Lewis CE, Schreiner PJ, Jacobs DR Jr, Sternfeld B, Sidney S. Joint associations of physical activity and aerobic fitness on the development of incident hypertension: coronary artery risk development in young adults. Hypertension 2010; 56(1):49-55.

17. Chei CL, Iso H, Yamagishi K, Tanigawa T, Cui R, Imano H, Kiyama M, Kitamura A, Sato S, Shimamoto T. Body fat distribution and the risk of hypertension and diabetes among Japanese men and women. Hypertens Res 2008; 31(5):851-857.

18. Costa JSD, Barcellos FC, Sclowitz ML, Sclowitz IKT, Castanheira M, Olinto MTA, Menezes AMB, Gigante DP, Macedo S, Fuchs SC. Prevalência de hipertensão arterial em adultos e fatores associados: um estudo de base populacional urbana em Pelotas, Rio Grande do Sul, Brasil. Arq Bras Cardiol 2007; 88(1):59-65.

19. Doğan N, Toprak D, Demir S. Hypertension prevalence and risk factors among adult population in Afyonkarahisar region: a cross-sectional research. Anadolu Kardiyol Derg 2012; 12(1):47-52.

20. Doulougou B, Kouanda S, Rossier C, Soura A, Zunzunegui MV. Differences in hypertension between informal and formal areas of Ouagadougou, a sub-Saharan African city. BMC Public Health 2014; 14:893.

21. Esteghamati A, Abbasi M, Alikhani S, Gouya MM, Delavari A, Shishehbor MH, Forouzanfar M, Hodjatzadeh A, Ramezani RD. Prevalence, awareness, treatment, and risk factors associated with hypertension in the Iranian population: the national survey of risk factors for noncommunicable diseases of Iran. Am J Hypertens 2008; 21(6):620-626.

22. Ferreira SRG, Moura EC, Malta DC, Sarno F. Freqüência de hipertensão arterial e fatores associados: Brasil, 2006. Rev Saude Publica 2009; 43(2):98-106.

23. Fujita M, Hata A. Sex and age differences in the effect of obesity on incidence of hypertension in the Japanese population: A large historical cohort study. J Am Soc Hypertens 2014; 8(1):64-70.

24. Harshfield E, Chowdhury R, Harhay MN, Bergquist $\mathrm{H}$, Harhay MO. Association of hypertension and hyperglycaemia with socioeconomic contexts in resource-poor settings: the Bangladesh Demographic and Health Survey. Int J Epidemiol 2015; 44(5):16251636.

25. Helelo TP, Gelaw YA, Adane AA. Prevalence and associated factors of hypertension among adults in Durame Town, Southern Ethiopia. Plos One 2014; 9(11):e112790.

26. Heraclides A, Mishra GD, Hardy RJ, Geleijnse JM, Black S, Prynne CJ, Kuh D, Soedamah-Muthu SS. Dairy intake, blood pressure and incident hypertension in a general British population: the 1946 birth cohort. Eur J Nutr 2012; 51(5):583-591. 
27. Hirani V, Zaninotto P, Primatesta P. Generalised and abdominal obesity and risk of diabetes, hypertension and hypertension-diabetes co-morbidity in England. Public Health Nutr 2008; 11(5):521-527.

28. Janghorbani M, Amini M, Gouya MM, Delavari A, Alikhani S, Mahdavi A. Nationwide survey of prevalence and risk factors of prehypertension and hypertension in Iranian adults. J Hypertens 2008; 26(3):419426.

29. Leenen FH, McInnis NH, Fodor G. Obesity and the prevalence and management of hypertension in Ontario, Canada. Am J Hypertens 2010; 23(9):1000-1006.

30. Lwin-Mm-Khin, Tassanee S, Oranut P, Chaweewon B. Risk factors for hypertension among rural Thais. Southeast Asian J Trop Med Public Health 2011; 42(1):2082017.

31. Malekzadeh MM, Etemadi A, Kamangar F, Khademi $\mathrm{H}$, Golozar A, Islami F, Pourshams A, Poustchi H, Navabakhsh B, Naemi M, Pharoah PD, Abnet CC, Brennan P, Boffetta P, Dawsey SM, Esteghamati A, Malekzadeh R. Prevalence, awareness and risk factors of hypertension in a large cohort of Iranian adult population. J Hypertens 2013; 31(7):1364-1371.

32. Muraro AP, Santos DF, Rodrigues PRM, Braga JU. Fatores associados à Hipertensão Arterial Sistêmica autorreferida segundo VIGITEL nas 26 capitais brasileiras e no Distrito Federal em 2008. Cien Saude Colet 2013; 18(5):1387-1398.

33. Niu J, Seo DC. Central obesity and hypertension in Chinese adults: a 12-year longitudinal examination. Prev Med 2014; 62:113-118.

34. Núñez-Córdoba JM, Valencia-Serrano F, Toledo E, Alonso A, Martínez-González M. The Mediterranean diet and incidence of hypertension: the Seguimiento Universidad de Navarra (SUN) Study. Am J Epidemiol 2009; 169(3):339-346.

35. Pang W, Sun Z, Zheng L, Li J, Zhang X, Liu S, Xu C, Li J, Hu D, Sun Y. Body mass index and the prevalence of prehypertension and hypertension in a Chinese rural population. Intern Med 2008; 47(10):893-897.

36. Radhika G, Sathya RM, Sudha V, Ganesan A, Mohan $\mathrm{V}$. Dietary salt intake and hypertension in an urban south Indian population [CURES - 53]. J Assoc Physicians India 2007; 55:405-411.

37. Sesso HD, Cook NR, Buring JE, Manson JE, Gaziano JM. Alcohol consumption and the risk of hypertension in women and men. Hypertension 2008; 51(4):1080-1087.

38. Shankarishan P, Borah PK, Mohapatra PK, Ahmed G, Mahanta J. Population attributable risk estimates for risk factors associated with hypertension in an Indian population. Eur J Prev Cardiol 2012; 20(6):963-971.

39. Silva DAS, Petroski EL, Peres MA. Pré-hipertensão e hipertensão em adultos de Florianópolis: estudo de base populacional. Rev Saude Publica 2012; 46(6):988998.

40. Song HJ, Paek YJ, Choi MK, Lee HJ. Gender differences in the relationship between risk of hypertension and fruit intake. Prev Med 2014; 67:154-159.
41. Tsubota-Utsugi M, Ohkubo T, Kikuya M, Metoki H, Kurimoto A, et AL. High fruit intake is associated with a lower risk of future hypertension determined by home blood pressure measurement: the OHASAMA study. J Hum Hypertens 2011; 25(3):164-171.

42. Sampson UK, Edwards TL, Jahangir E, Munro H, Wariboko M, Wassef MG, Fazio S, Mensah GA, Kabagambe EK, Blot WJ, Lipworth L. Factors associated with the prevalence of hypertension in the southeastern United States: insights from 69,211 blacks and whites in the Southern Community Cohort Study. Circ Cardiovasc Qual Outcomes 2014; 7(1):33-54.

43. Veenstra G. Race, gender, class, sexuality (RGCS) and hypertension. Soc Sci Med 2013; 89:16-24.

44. Camacho PA, Gomez-Arbelaez D, Molina DI, Sanchez G, Arcos E, Narvaez C, García H, Pérez M, Hernandez EA, Duran M, Cure C, Sotomayor A, Rico A, David TM, Cohen DD, Rangarajan S, Yusuf S, Lopez-Jaramillo P. Social disparities explain differences in hypertension prevalence, detection and control in Colombia. J Hypertens 2016; 34(12):2344-2352.

45. Liu X, Gu W, Li Z, Lei H, Li G, Huang W. Hypertension prevalence, awareness, treatment, control and associated factors in Southwest China: an update. $J$ Hypertens 2017; 35(3):637-644.

46. Malta DC, Bernal RTI, Andrade SSCA, Silva MMA, Velasquez-Melendez G. Prevalência e fatores associados com hipertensão arterial autorreferida em adultos brasileiros. Rev Saude Publica 2017; 51(Supl. 1):11s.

47. Moreira APL, Malta DC, Vianna RPT, Moreira PVL, Carvalho AT. Risk and protection factors for self-reported hypertension and diabetes in João Pessoa, Brazil. The VIGITEL survey, 2014. A cross-sectional study. Sao Paulo Med J 2017; 135(5):450-461.

48. Oliveira CM, Ulbrich AZ, Neves FS, Dias FAL, Horimoto ARVR, Krieger JE, Alvim RO, Pereira ADC. Association between anthropometric indicators of adiposity and hypertension in a Brazilian population: Baependi Heart Study. PLoS One 2017; 12(10):e0185225.

49. Silva RC, Silva DA, Bastos JL, Peres KG, Peres M, González-Chica DA. Anthropometric measures change and incidence of high blood pressure levels among adults: a population-based prospective study in Southern Brazil. J Hypertens 2017; 35(1):39-46.

50. Soubeiga JK, Millogo TB, Bicaba BW, Doulougou B, Kouanda S. Prevalence and factors associated with hypertension in Burkina Faso: a countrywide crosssectional study. BMC Public Health 2017; 17(1):64.

51. Malta DC, Bernal RT, Souza MF, Szwarcwald CL, Lima MG, Barros MB. Social inequalities in the prevalence of self-reported chronic non-communicable diseases in Brazil: national health survey 2013. Int J Equity Health 2016; 15(1):153.

52. Miranda RD, Perrotti TC, Bellinazzi VR, Nóbrega TM, Cendoroglo MS, Toniolo Neto J. Hipertensão arterial no idoso: peculiaridades na fisiopatologia, no diagnóstico e no tratamento. Rev Bras Hipertens 2002; 9(3):293-300. 
53. The Lancet. Hypertension: an urgent need for global control and prevention. Lancet 2014; 383(9932):1861.

54. Andrade SSA, Stopa SR, Brito AS, Chueri OS, Szwarcwald CL, Malta DC. Prevalência de hipertensão arterial autorreferida na população brasileira: análise da Pesquisa Nacional de Saúde, 2013. Epidemiol Serv Saude 2015; 24(2):297-304.

55. Barros MBA, Francisco PMSB, Zanchetta LM, César CLG. Tendências das desigualdades sociais e demográficas na prevalência de doenças crônicas no Brasil, PNAD: 2003-2008. Cien Saude Colet 2011; 16(9):3755-3768.

56. Malta DC, Santos NB, Perillo RD, Szwarcwald CL. Prevalence of high blood pressure measured in the Brazilian population, National Health Survey, 2013. Sao Paulo Med J 2016; 134 (2):163-170.

57. Travassos CMR, Viacava F, Pinheiro RS, Brito AS. Utilização dos serviços de saúde no Brasil: gênero, características familiares e condição social. Rev Panam Salud Publica 2002; 11(5-6):365-373.

58. Cooper RS, Rotimi CN, Ward R. The puzzle of hypertension in African-Americans. Sci Am 1999; 280(2):56-63.

59. Danziger RS. Hypertension in an anthropological and evolutionary paradigm. Hypertension 2001; 38(1):1922.

60. Kaufman JS, Hall SA. The slavery hypertension hypothesis: dissemination and appeal of a modern race theory. Epidemiology 2003; 14(1):111-118.

61. Laguardia J. Raça, genética \& hipertensão: nova genética ou velha eugenia? Hist Cien Saude Manguinhos 2005; 12(2):371-393.

62. Fan AZ, Strasser SM, Zhang X, Fang J, Crawford CG. State socioeconomic indicators and self- reported hypertension among US adults, 2011 behavioral risk factor surveillance system. Prev Chronic Dis 2015; 12:E27.

63. Grotto I, Huerta M, Sharabi Y. Hypertension and socioeconomic status. Curr Opin Cardiol 2008; 23(4):335-339.

64. NCD Risk Factor Collaboration (NCD-RisC). Trends in adult body-mass index in 200 countries from 1975 to 2014: a pooled analysis of 1698 population-based measurement studies with 19.2 million participants. Lancet 2016; 387(10026):1377-1396.

65. Instituto Brasileiro de Geografia e Estatística (IBGE) Pesquisa Nacional de Saúde, 2013: percepção do estado de saúde, estilos de vida e doenças crônicas. Rio de Janeiro: IBGE; 2014.
66. Chen L, Smith GD, Harbord RM, Lewis SJ. Alcohol intake and blood pressure: a systematic review implementing a Mendelian randomization approach. PLoS Med 2008; 5(3):e52.

67. Nunes E. Consumo do tabaco. Efeitos na saúde. Rev Port Clin Geral 2006; 22(2):225-244.

68. Groppelli A, Giorgi DM, Omboni S, Parati G, Mancia G. Persistent blood pressure increase induced by heavy smoking. J Hypertens 1992; 10(5):495-499.

69. Mathers CD, Loncar D. Projections of global mortality and burden of disease from 2002 to 2030. PLoS Med 2006; 3(11):e442.

70. Lima-Costa MF, Peixoto SV, Firmo JOA. Validade da hipertensão arterial auto-referida e seus determinantes (projeto Bambuí). Rev Saude Publica 2004; 38(5):637-642.

71. Singh GK, Daus GP, Allender M, Ramey CT, Martin EK, Perry C, Reyes AAL, Vedamuthu IP. Social Determinants of Health in the United States: Addressing Major Health Inequality Trends for the Nation, 19352016. Int J MCH AIDS 2017; 6(2):139-164.

72. GBD 2015 Risk Factors Collaborators. Global, regional, and national comparative risk assessment of 79 behavioral, environmental and occupational, and metabolic risks or clusters of risks, 1990-2015: a systematic analysis for the Global Burden of Disease Study 2015. Lancet 2016; 388(10053):1659-1724

73. Szwarcwald CL, Montilla DER, Marques AP, Damacena GN, Almeida WS, Malta DC. Inequalities in healthy life expectancy by Federated States. Rev Saude Publica 2017; 51(Supl. 1):7s.

Artigo apresentado em 14/06/2018

Aprovado em 03/10/2018

Versão final apresentada em 05/10/2018 